\title{
Increased SPC24 in prostatic diseases and diagnostic value of SPC24 and its interacting partners in prostate cancer
}

\author{
SUIXIA CHEN ${ }^{1-3^{*}}$, XIAO WANG ${ }^{1,3 *}$, SHENGFENG ZHENG ${ }^{1,4^{*}}$, HONGWEN LI ${ }^{5}$, \\ SHOUXU QIN ${ }^{1,3}$, JIAYI LIU ${ }^{1,6}$, WENXIAN JIA ${ }^{1}$, MENGNAN SHAO ${ }^{1}$, YANJUN TAN ${ }^{1,2}$, \\ HUI LIANG ${ }^{1}$, WEIRU SONG ${ }^{7}$, SHAOMING LU $^{8}$, CHENGWU LIU $^{3}$ and XIAOLI YANG ${ }^{1,2}$ \\ ${ }^{1}$ Scientific Research Center, Guilin Medical University; ${ }^{2}$ Guangxi Health Commission Key Laboratory of Disease \\ Proteomics Research, Guilin Medical University, Guilin, Guangxi 541100; ${ }^{3}$ Department of Pathophysiology, \\ Guangxi Medical University; ${ }^{4}$ Department of Urology, The First Affiliated Hospital of Guangxi Medical University, \\ Nanning, Guangxi 530021; ${ }^{5}$ Department of Anatomy, Faculty of Basic Medical Sciences, Guilin Medical University, \\ Guilin, Guangxi 541100; Departments of ${ }^{6}$ Pathology and ${ }^{7}$ Andrology, The First Affiliated Hospital of \\ Guangxi Medical University, Nanning, Guangxi 530021; ${ }^{8}$ Center for Reproductive Medicine, \\ Shandong Provincial Hospital Affiliated to Shandong University, Jinan, Shandong 250200, P.R. China
}

Received August 25, 2019; Accepted March 17, 2021

DOI: $10.3892 / \mathrm{etm} .2021 .10355$

\begin{abstract}
SPC24 is a crucial component of the mitotic checkpoint machinery in tumorigenesis. High levels of SPC24 have been found in various cancers, including breast cancer, lung cancer, liver cancer, osteosarcoma and thyroid cancer. However, to the best of our knowledge, the impact of SPC24 on prostate cancer ( $\mathrm{PCa}$ ) and other prostate diseases remains unclear. In the present study expression of global SPC24 messenger RNA (mRNA) was assessed in a subset of patients with PCa included in The Cancer Genome Atlas (TCGA) database. Increased levels of SPC24 expression were found in PCa patients $>60$ years old compared to patients $<60$ and increased SPC24 expression was also associated with higher levels of prostate specific antigen $(\mathrm{P}<0.05)$ and lymph node metastasis $(\mathrm{P}<0.05)$. Higher levels of SPC24 expression were associated with negative outcomes in $\mathrm{PCa}$ patients $(\mathrm{P}<0.05)$. Furthermore, in Chinese patients with prostatitis, benign prostatic hypertrophy (BPH) and PCa, SPC24 was expressed at significantly higher levels than that in adjacent/normal tissues, as assessed by reverse transcription-quantitative polymerase
\end{abstract}

Correspondence to: Professor Chengwu Liu, Department of Pathophysiology, Guangxi Medical University, Building 102, 22 Shuangyong Road, Nanning, Guangxi 530021, P.R. China

E-mail: chengwuliu@163.com

Professor Xiaoli Yang, Scientific Research Center, Guilin Medical University, 1 Zhiyuan Road, Guilin, Guangxi 541100, P.R. China

E-mail: cncsyxl@126.com

*Contributed equally

Key words: SPC24, prostatitis, benign prostatic hypertrophy, prostate cancer, biomarker, diagnosis chain reaction, immunohistochemistry and western blotting. High expression of SPC24 was associated with high Gleason stages (IV and V; P<0.05). Further analysis, based on Gene Ontology and pathway functional enrichment analysis, suggested that nuclear division cycle 80 (NDC80), an SPC24 protein interaction partner, and mitotic spindle checkpoint serine/threonine-protein kinase BUB1 (BUB1), a core subunit of the spindle assembly checkpoint, may be associated with SPC24 in PCa development. Finally, using binary logistic regression, algorithms combining the receiver operating characteristic between SPC24 and BUB1 or NDC80 indicated that a combination of these markers may provide better PCa diagnosis ability than other PCa diagnosis markers. Taken together, these findings suggest that SPC 24 may be a promising prostate disease biomarker.

\section{Introduction}

SPC24 is a component of the nuclear division cycle 80 (NDC80) kinetochore complex and is involved in the formation of the kinetochore complex along with three other proteins, NUF2, NDC80 and SPC25 (1). The NDC80 kinetochore complex mediates microtubule binding and is anchored onto the inner kinetochore via the SPC24-SPC25 protein complex. In addition, the SPC24-SPC25 protein complex mediates dynamic interactions between nuclear spindle microtubules and kinetochores, which ensures faithful and accurate chromosomal segregation during mitosis $(2,3)$. Abnormal mitosis is a common hallmark of cancer (4). It has also been reported that mutation of SPC24 may lead to a chromosome segregation defect (5). Moreover, simultaneous disruption of both SPC24 and SPC25 may cause a spindle checkpoint defect in the cell, allowing the cell to bypass mitosis (6). These findings suggest that dysregulation of SPC24 may lead to genomic instability and disrupt control of the cell cycle, which can ultimately promote malignancy. 
Prostate cancer (PCa) has been identified as the second most common cause of malignant tumor driven death in men (7). Dietary factors, lifestyle-related factors, region and genetic polymorphism have been recognized as contributors to the risk of $\mathrm{PCa}(8,9)$. Specific risk factors, such as prostatitis (inflammation/swelling of the prostate gland) and benign prostatic hypertrophy (BPH; proliferation of the cellular elements of the prostate, leading to an enlarged prostate gland) have been reported to have a strong association with the development of prostate cancer and a higher relative risk of prostate cancer was estimated in men with both of these conditions (10). BPH has shown a high correlation with prostate cancer in Asian populations (11). There is a $30 \%$ probability of biochemical relapse [prostate specific antigen (PSA) $<0.2 \mathrm{ng} / \mathrm{ml}$ twice in a row with no recurrent or metastatic lesions found on radiography] in prostate cancer patients within the first 5 years after radical prostatectomy (12). In the clinic, detection of PSA is often used for early diagnosis of prostate cancer. However, PSA is not specific to malignant $\mathrm{PCa}$ and has a high false-positive and false-negative rate of $\sim 15 \%$ (13). Therefore, a better PCa biomarkerwould be beneficial for accurate diagnosis.

High levels of SPC24 have been found to be expressed in various cancers, including breast, lung, thyroid and liver cancer and osteosarcoma (14-17). A recent study also indicated that increased SPC24 expression is associated with advanced stage lung tumors (15). An additional study indicated that when endogenous SPC24 was knocked down, the growth and invasion ability of anaplastic thyroid cancer cells was inhibited and cell apoptosis was promoted (16). However, the role played by $\mathrm{SPC} 24$ in $\mathrm{PCa}$ is unclear.

In the present study, RNA-Seq data from The Cancer Genome Atlas (TCGA) database were used to analyze SPC24 messenger RNA (mRNA) levels in PCa tissues and normal tissues. Subsequently, reverse transcription-quantitative polymerase chain reaction (RT-qPCR) and western blot analysis were conducted to measure SPC24 expression in $\mathrm{PCa}$ and adjacent tissues, along with BPH tissues. SPC24 expression was also detected in 40 clinical BPH tissues, 35 PCa tissues, 35 paired adjacent tissues, 12 prostatitis tissues and 9 normal prostate tissues by immunohistochemistry (IHC). In these tissues, the association of SPC24 with clinicopathological features was also assessed. Gene ontology (GO) analyses, Reactome pathway analyses and Kyoto Encyclopedia of Genes and Genomes (KEGG) pathway analysis of the identified genes were conducted to find correlations between SPC24 and its interactors in biological processes, which were confirmed by qPCR.

\section{Materials and methods}

Data collection and analysis of TCGA database. RNA sequencing (RNA-seq) data from selected genes (SPC24 and its interacting partners obtained from literature review and online database prediction) in $\mathrm{PCa}$ and clinicopathological information of patients were downloaded from the TCGA Prostate Cancer (PRAD) database (https://xena.ucsc. edu/public/; version 2017-10-13). This dataset indicates the gene-level transcription estimates, as $\log 2(x+1)$ transformed RSEM (RNA-Seq by expectation-maximization) normalized counts. The 3rd level data (normalized and quantified raw data) was obtained from the TCGA data coordination center (cancer.gov/about-nci/organization/ccg/research/structural-genomics/tcga).

Patients and specimens. From 2016 to 2018, a total of 96 patients (age, 46-87 years) including 35 patients with $\mathrm{PCa}$ (median age, 65 years), 40 patients with BPH (median age, 64 years), 12 patients with prostatitis (median ages, 68 years) and 9 patients without prostate disease (median age, 62 years) participated in the present study. Written informed consent from patients and permission from the Ethics Committee of The First Affiliated Hospital of Guangxi Medical University were acquired. No preoperative treatment was performed before surgery. All samples were provided by the First Affiliated Hospital of the Guangxi Medical University. BPH tissues were collected from patients with transurethral plasmakinetic resection of prostate. PCa tissues and adjacent tissues were obtained from patients that underwent radical prostatectomy, while prostatitis tissues and normal tissues were obtained from patients that underwentradical cystectomy. All prostate samples with hematoxylin and eosin staining (H\&E) were diagnosed by two expert pathologists. Tumor grades were calculated in accordance with Gleason classification. Clinical features of patients including ethnicity, age, smoking, drinking, PSA, urea, creatinine, lithic acid, $\mathrm{HCO}_{3}{ }^{-}$, and creatinine clearance $(\mathrm{CrCl})$ were obtained by Medical History Taking, PSA Test, Blood Gas Test, and Renal Panel.

Quantitative real-time polymerase chain reaction. TRIzol ${ }^{\circledR}$ reagent (Invitrogen; Thermo Fisher Scientific, Inc.) was used to extract total RNA from the clinical BPH, PCa and adjacent tissues. A NanoDrop spectrophotometer (Thermo Fisher Scientific, Inc.) was used to assess the purity and quantity of the isolated RNA. Complementary DNA (cDNA) was synthesized using the Applied Biosystems StepOne (Thermo Fisher Scientific, Inc.) with qPCR kit and SYBR-Green master mix (Roche Diagnostics.). According to the instructions of the RT kit (PrimeScript ${ }^{\mathrm{TM}}$ RT Master Mix; Takara), RT was performed as follows: Initiation at $37^{\circ} \mathrm{C}$ for $15 \mathrm{~min}$, then $85^{\circ} \mathrm{C}$ for $5 \mathrm{sec}$. The qPCR conditions included an initiation at $95^{\circ} \mathrm{C}$ for $10 \mathrm{~min}$, followed by 40 cycles of $95^{\circ} \mathrm{C}$ for $15 \mathrm{sec}$ and $60^{\circ} \mathrm{C}$ for $1 \mathrm{~min}$. The $2^{-\Delta \Delta \mathrm{Cq}}$ method (18) was performed to analyze relative quantities for the level of mRNA expression. The specific primer sequences were as follows: 5'-TGGCCTCAG CTAGGTAACCA-3' (forward) and 5'-GTACCTGGGAGC TGTCATCG-3' (reverse) for SPC24. 5'-GAAGCGCAGTTC AGTTTCC-3' (forward) and 5'-GGTTTCTCTTTGGTTTGA GGG-3' (reverse) for NDC80. 5'-TGGGAAAGATACATA CAGTGG-3' (forward) and 5'-GAATCTTGGGTCATTGTG GT-3' (reverse) for BUB1.

Western blot analysis. Clinical PCa tissues and paired adjacent tissues were obtained, washed twice with 0.01 M PBS and lysed on ice with radioimmunoprecipitation assay (RIPA, Beijing Solarbio Science \& Technology Co., Ltd.) a cell lysis reagent for $30 \mathrm{~min}$. Tissues were centrifuged at $16,000 \mathrm{x} \mathrm{g}$ for $15 \mathrm{~min}$ at $4^{\circ} \mathrm{C}$ to remove insoluble proteins. The concentration of extracted protein was determined by bicinchoninic acid (BCA) protein assay kit (Leagene, Inc.). A total of $50 \mu \mathrm{g}$ of each total protein extract was separated using $10 \%$ sodium 
dodecyl sulfate-polyacrylamide gel electrophoresis and transferred onto PVDF membranes (Bio-Rad Laboratories, Inc.). After being blocked at $23^{\circ} \mathrm{C}$ for $1 \mathrm{~h}$ with $5 \%$ milk solution, membranes were washed three times with Tris-buffered saline-tween $(0.1 \%$ Tween-20) solution. The membranes were then incubated with primary antibodies, rabbit anti-human SPC24 (Abcam; 1:1,000; cat. no. ab169786) and $\beta$-actin (Proteintech Group, Inc.; 1:1,000; cat. no. HRP-60008) at $4^{\circ} \mathrm{C}$ overnight. After being washed with TBST, the membranes were incubated with horseradish peroxidase-labeled goat-anti-rabbit IgG secondary antibody (Abcam; 1:4,000; cat. no. ab6721) at $37^{\circ} \mathrm{C}$ for $1 \mathrm{~h}$. The membranes were processed for protein detection using the BeyoECL Plus Kit (Beyotime Institute of Biotechnology).

Immunohistochemistry. All tissues were embedded in paraffin and then cut into $4-\mu$ m-thick sections. Immunostaining was conducted using the Elivision $^{\mathrm{TM}}$ Plus method (EliVision ${ }^{\mathrm{TM}}$ Super KIT9922; Fuzhou Maixin Biotech. Co., Ltd.), with the procedure performed according to the kit instructions. All samples were deparaffinized with xylene and rehydrated with a graded ethanol series (100, 95, 90 and 85\%). Citrate buffer ( $\mathrm{pH}$ 6.0) was used for antigen retrieval. Methanol containing $3 \% \mathrm{H}_{2} \mathrm{O}_{2}$ solution was used to block endogenous peroxidase activity (at $23^{\circ} \mathrm{C}$ for $15 \mathrm{~min}$ without light), before $10 \%$ goat serum (OriGene Technologies, Inc.) was used to block nonspecific proteins (at $23^{\circ} \mathrm{C}$ for $15 \mathrm{~min}$ ). Anti-SPC24 antibody (cat. no. ab169786) primary antibody was added at a dilution of 1:250 and the sections were subsequently incubated overnight at $4^{\circ} \mathrm{C}$. After washing in PBS, all sections were incubated with biotinylated secondary antibodies (1:100, cat. no. SP-9000; OriGene Technologies, Inc.) at $23^{\circ} \mathrm{C}$ for $25 \mathrm{~min}$. All sections were stained with horseradish-labeled chains of ovalbumin working fluid (OriGene Technologies, Inc.) at $23^{\circ} \mathrm{C}$ for $15 \mathrm{~min}$, then were washed in PBS for 5 min (repeated 3 times). The images were allowed to develop in diaminobenzidine DAB substrate Kit (ZLI9018; Beijing Zhongshan Golden Bridge Biotechnology Co., Ltd.). Finally, all sections were re-dyed with hematoxylin for $1 \mathrm{~min}$ at $23^{\circ} \mathrm{C}$ and mounted with neutral resin. The degree of immunohistochemistry (IHC) staining was evaluated by two independent blinded pathologists (light microscope; cat. no. BX53+DP80; Olympus Corporation) observing $\geq 20$ fields of view in each slide. The results were analyzed by an immunoreactivity score (IRS) system based on staining intensity and cell staining proportion data. The staining intensity was scored from $0-3$ as follows: $0=$ unstained; $1=$ weakly stained; $2=$ moderately stained and $3=$ strongly stained. The proportion of cell staining was scored on a scale from $0-4$ as follows: $0=$ negative; $1=1-10 \% ; 2=11-50 \%$; $3=51-80 \%$ and $4>80 \%$. The intensity score was multiplied by the proportion of staining to obtain an immunoreactivity score. A total score $>3$ was considered to be a high level of expression. A total score of 1-3 was considered as low expression and 0 was considered no expression.

Functional and pathway enrichment analysis. To analyze the identified genes at the functional level, Gene ontology (GO) analyses, Reactome pathway analyses (reactome.org/) and Kyoto Encyclopedia of Genes and Genomes (KEGG) pathway analyses of the identified genes were conducted. The biological online database tool adopted in this study for $\mathrm{GO}$ and KEGG analysis was the Search Tool for the Retrieval of Interacting Genes (STRING, Version: 11.0, https://string-db. org/). Identified genes were submitted and Homo sapiens was selected in the species column. Finally, the GO terms or KEGG pathways with the cut-off criteria $(\mathrm{P}<0.05)$ were identified to be associated with the input genes.

Statistical analysis. Software including SPSS 21.0 (IBM Corp.), GraphPad Prism 5 (Graph Pad Software, Inc.) and MedCalc. v9.2.0.1 (MedCalc, Inc.) were used to perform statistical analyses. Unpaired t-tests were used to compare quantitative sample data from the TCGA. Data are presented in the form of the mean \pm SD. Two paired-group (PCa/BPH/prostatitis tissues and adjacent/normal tissues) comparisons were conducted using $\chi^{2}$ test with Fisher's exact test. Association between SPC24 expression and clinicopathological factors was also determined using $\chi^{2}$ test. A P-value of $<0.05$ was considered statistically significant. Overall survival (OS) and Disease-free survival (DFS) times were stratified according to median expression level (high and low) of SPC24 (conducted in GEPIA version 2017, http:/gepia.cancer-pku. $\mathrm{cn} /$ ). Survival analyses were conducted and visualized using Kaplan-Meier analysis and log-rank test. The diagnostic ability was evaluated using receiver operating characteristic curve (ROC). The sensibility and specificity were obtained at an optimal cut-off with the max Youden index. Based on mRNA data (quantified as RSEM to estimate gene and isoform expression levels from RNA-Seq data; deweylab. github.io/RSEM/README.html) extracted from TCGA database, diagnostic ability of two combined models was also analyzed by ROC curves using binary logistic regression. Combined models acquired using binary logistic regression were: SPC24 + BUB1: Y=SPC24+BUB1*0.849/1.230; SPC24 + NDC80: Y=SPC24+NDC80*(-1.022)/1.486

\section{Results}

SPC24 analyses in TCGA dataset. The expression of SPC24 in $\mathrm{PCa}$ was significantly higher than that in normal tissues ( $\mathrm{P}<0.0001$; Fig. 1A). In addition, higher SPC24 expression was found in older PCa patients ( $>60)$, higher Gleason scores (8-10), and with higher PSA values ( $>0.1$; Table I). Increased SPC24 was also associated with lymph node metastasis (Table I). An ROC curve was plotted to further evaluate the diagnostic value of SPC24 and the AUC value of SPC24 was $0.821(\mathrm{P}<0.05)$. Based on the maximum Youden index discriminating patients with PCa from controls, an optimal cut-off value of 2.06 was obtained, and its sensitivity and specificity for predicting $\mathrm{PCa}$ were calculated to be 86.54 and $67.07 \%$, respectively (Fig. 1B). Survival analyses also suggested that high SPC24 expression was associated with negative outcomes in $\mathrm{PCa}$ patients $(\mathrm{P}<0.05$ : Fig. 1C and D).

Expression of SPC24 in BPH, PCa, prostatitis and adjacent/normal tissues. To further confirm the expression of SPC24 in PCa, qPCR, western blot and IHC were performed in $35 \mathrm{PCa}$ tissues and paired adjacent tissues from Chinese patients with PCa. Increased SPC24 expression was found in $\mathrm{PCa}$ tissues compared with adjacent tissues $(\mathrm{P}<0.05$ : 
Table I. Association between SPC24 and clinicopathologic characteristics in The Cancer Genome Atlas PRAD database (n=498).

\begin{tabular}{|c|c|c|c|}
\hline Clinical feature & Number of cases & SPC24 (RSEM; mean \pm SD) & P-value \\
\hline Sample & & & $<0.05^{\mathrm{a}}$ \\
\hline $\mathrm{PCa}$ & 498 & $2.590 \pm 1.179$ & \\
\hline Normal & 52 & $1.303 \pm 0.928$ & \\
\hline Age at diagnosis (years) & & & $<0.05^{\mathrm{a}}$ \\
\hline$\leq 60$ & 223 & $2.405 \pm 1.128$ & \\
\hline$>60$ & 275 & $2.740 \pm 1.200$ & \\
\hline Gleason score & & & $<0.05^{\mathrm{a}}$ \\
\hline $2-4$ & 0 & & \\
\hline $5-7$ & 292 & $2.247 \pm 0.969$ & \\
\hline $8-10$ & 206 & $3.077 \pm 1.277$ & \\
\hline Laterality & & & $>0.05$ \\
\hline Bilateral & 433 & $2.599 \pm 1.193$ & \\
\hline Unilateral & 65 & $2.534 \pm 1.083$ & \\
\hline PSA value & & & $<0.05^{\mathrm{a}}$ \\
\hline$\leq 0.1$ & 329 & $2.456 \pm 1.125$ & \\
\hline$>0.1$ & 112 & $2.824 \pm 1.24$ & \\
\hline Unknown & 27 & & \\
\hline Tumor level & & & $>0.05$ \\
\hline Apex & 292 & $2.66 \pm 1.181$ & \\
\hline MiddlelBase & 63 & $2.588 \pm 1.153$ & \\
\hline Unknown & 143 & & \\
\hline Lymph node & & & $<0.05^{\mathrm{a}}$ \\
\hline No & 345 & $2.533 \pm 1.122$ & \\
\hline N1 & 80 & $3.103 \pm 1.313$ & \\
\hline Unknown & 73 & & \\
\hline \multicolumn{4}{|l|}{ Metastasis } \\
\hline M0 & 456 & & \\
\hline M1 & 3 & & \\
\hline Unknown & 39 & & \\
\hline
\end{tabular}

PCa, prostate cancer; PSA, prostate specific antigen; RSEM, RNA-Seq by Expectation-Maximization; standard deviation. ${ }^{\mathrm{a} P}<0.05$.

Figs. 2 and 3). qPCR analysis also suggested that SPC24 expression was higher in $\mathrm{PCa}$ than in $\mathrm{BPH}$ and adjacent tissues $(\mathrm{P}<0.05$; Fig. 2A). IHC (Fig. 3B) indicated that 13 of the 35 PCa patients had high SPC24 expression, while the remaining 22 had low expression. Of the $40 \mathrm{BPH}$ patients, 11 had high SPC24 expression and the remaining 29 had low or no expression. Additionally, 5 of the 12 patients with prostatitis had high levels of SPC24 expression, while the remaining had no expression. SPC24 was expressed at low levels or not expressed in both the adjacent tissues (35/35) and the normal tissues (9/9; $\mathrm{P}<0.05$; Fig. 3B; Table II). These results indicated that SPC24 expression was higher in PCa tissues than in adjacent tissues, which was also confirmed by western blot (Fig. 3A). Higher SPC24 levels in BPH tissues than in the adjacent tissues were also found, while there was no difference between $\mathrm{PCa}$ and $\mathrm{BPH}$ in SPC24 expression (Fig. 3; Table II).

Association between SPC24 expression and clinical features of patients with $\mathrm{PCa} / \mathrm{BPH} /$ prostatitis. In Chinese patients with $\mathrm{PCa}$, high expression of SPC24 was associated with high Gleason stage (IV and V; $\mathrm{P}<0.05$ ). Other clinical features in $\mathrm{PCa}$, including ethnicity, age, smoking, drinking, PSA, urea, creatinine, lithic acid, $\mathrm{HCO}_{3}{ }^{-}$, and creatinine clearance, were not correlated with SPC24 expression (Table III). In BPH tissues, SPC24 levels were associated with ethnicity and $\mathrm{CrCl}$ $(\mathrm{P}<0.05$; Table IV). However, there was no association between SPC24 and other clinical factors in prostatitis.

Gene ontology and pathway functional enrichment analysis. As shown in Table V, GO annotation and pathway enrichment analysis suggested that SPC24 and its interactors were related to several biological processes, including cell division and cell cycle. In addition, SPC24 and its interactors were related to terms suggesting they were located in the nucleus, cytosol and intracellular organelle components of the cell. Reactome pathway analysis indicated that SPC24 participated in many pathways, as it was associated with the terms resolution of sister chromatid cohesion, amplification 
A

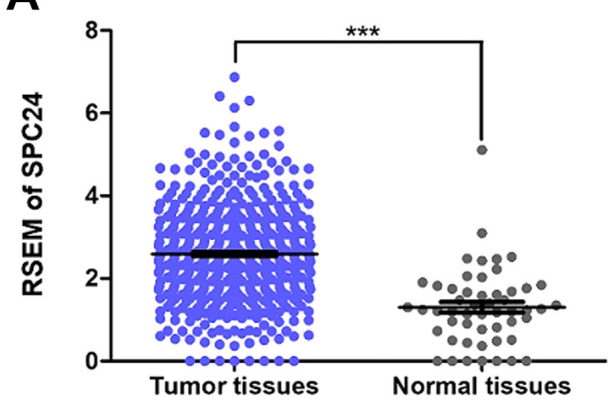

C

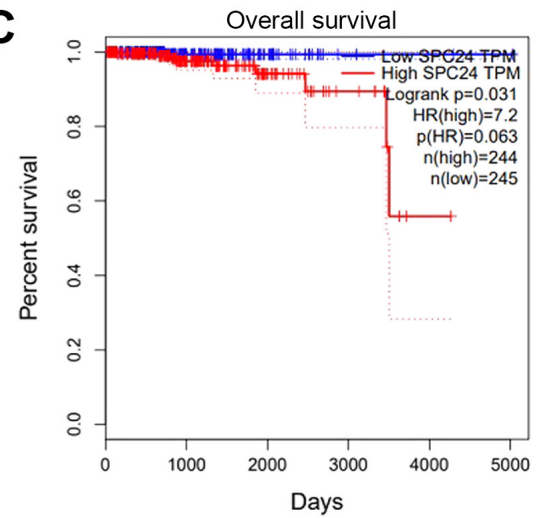

B
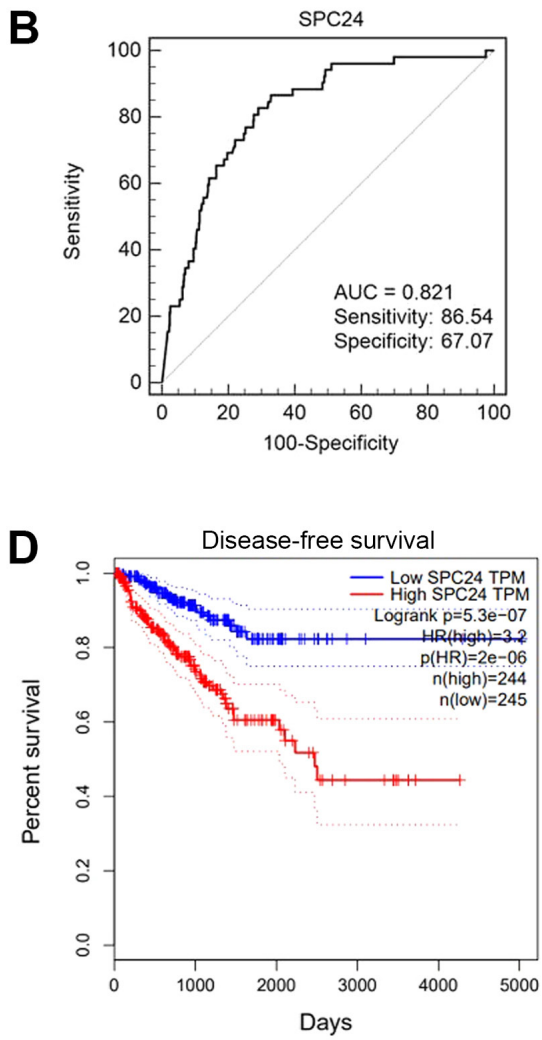

Figure 1. Data mining of SPC24 in The Cancer Genome Atlas PRAD database. (A) Increased SPC24 levels were seen in PCa tissues compared to normal tissues $\left({ }^{* * *} \mathrm{P}<0.0001\right)$. (B) ROC of SPC24 in diagnosis of PCa (C) OS and (D) DFS time of PCa patients with low/high expression of SPC24. AUC, area under the curve; HR, hazard ratio; PCa, prostate cancer; OS, overall survival; DFS, disease free survival; ROC, receiver operator characteristic; RSEM, RNA-Seq by expectation-maximization.

A

SPC24

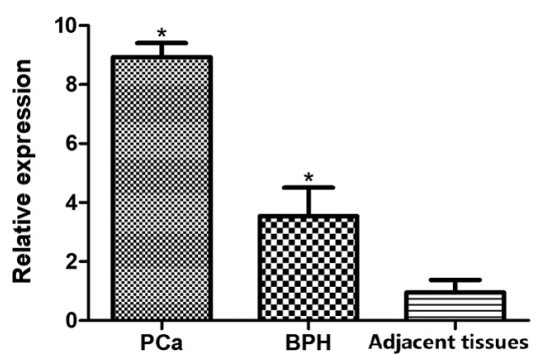

B

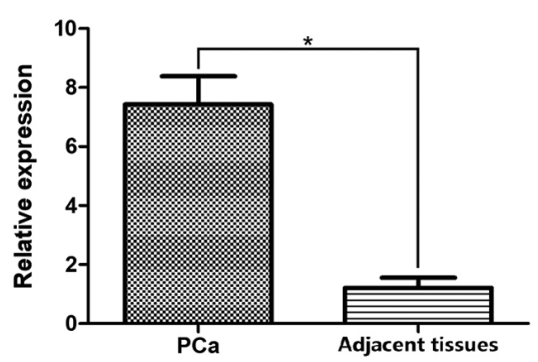

C

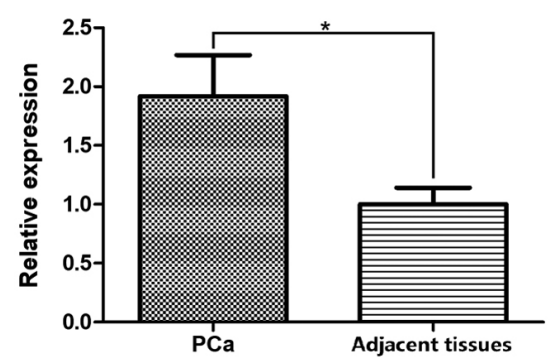

Figure 2. mRNA expression of SPC24 and its interactors in different prostate samples assessed by reverse transcription-quantitative PCR. (A) Higher expression of SPC24 in PCa and BPH tissues than that in adjacent tissues ( $\left.{ }^{*} \mathrm{P}<0.05\right)$. High expression of (B) NDC 80 and (C) BUB1 was found in PCa tissues compared with adjacent tissues ("P<0.05) PCa, prostate cancer; BPH, benign prostatic hypertrophy; BUB1, mitotic checkpoint serine threonine protein kinase BUB1.

of signal from unattached kinetochores via a MAD2 inhibitory signal, cell cycle checkpoints, RHO GTPases activate formins, separation of sister chromatids and signal transduction. The protein-protein interaction (PPI) network is shown in Fig. 4.

Increased expression of SPC24 interactors. In PCa samples, qPCR results showed that expression of SPC24 interacting proteins (BUB1 and NDC80) was higher than that in the adjacent tissues (Fig. 2B and C). Based on the mRNA data from TCGA database, two combined models via SPC24 were built to obtain better diagnosis efficiency for PCa using binary logistic regression (Fig. 5). The ROC curve of combined model of SPC24 and BUB1 was generated, and the sensitivity and specificity were 88.2 and $80.8 \%$, respectively. The AUC was 0.894 . The ROC curve of combined model of SPC24 and NDC80 had a AUC of 0.905 and the sensitivity and specificity were 84.7 and $96.2 \%$, respectively (Table VI).

\section{Discussion}

As a subunit of the NDC80 complex, NUF2 is highly expressed in a variety of tumors and has also been shown to correlate with poor prognosis $(19,20)$. It has been reported that SPC24 and SPC25 are required to establish and maintain kinetochore-microtubule attachments and metaphase alignment and are essential for chromosomal movement to the spindle poles during anaphase (21). High expression of SPC25 
Table II. Expression of SPC24 in PCa tissues, adjacent tissues, BPH tissues, prostatitis tissues, and normal prostate tissues.

A, Benign prostatic hypertrophy and adjacent tissues

\begin{tabular}{lccr}
\hline & & \multicolumn{2}{c}{ SPC24 level } \\
\cline { 3 - 4 } Clinical feature & Number of cases & Low/negative & High \\
\hline Disease tissue & 40 & 29 & 11 \\
Adjacent tissue & 35 & 35 & $0.001^{\mathrm{a}}$ \\
\hline
\end{tabular}

B, Prostate cancer tissues and adjacent tissues

\begin{tabular}{lcccr}
\hline & & \multicolumn{2}{c}{ SPC24 level } & \\
\cline { 3 - 4 } Clinical feature & Number of cases & Low/negative & High & P-value \\
\hline Disease tissue & 35 & 22 & 13 & $<0.001^{\text {a }}$ \\
Adjacent tissue & 35 & 35 & 0 & \\
\hline
\end{tabular}

C, Prostate cancer tissue and benign prostatic hypertrophy tissues

SPC24 level

\begin{tabular}{lcccr} 
Clinical feature & Number of cases & Low/negative & High & P-value \\
\hline PCa tissue & 35 & 22 & 13 & 0.459 \\
BPH tissue & 40 & 29 & 11 & \\
\hline
\end{tabular}

$\mathrm{D}$, Prostatitis tissue and normal tissue

\begin{tabular}{lccc}
\hline & & SPC24 level & \\
\cline { 3 - 4 } Clinical feature & Number of cases & Low/negative & High \\
\hline Prostatitis tissue & 12 & 7 & 5 \\
Normal tissue & 9 & 9 & 0 \\
\hline
\end{tabular}

BPH tissues were collected from patients undergoing transurethral plasmakinetic resection of prostate. PCa tissues and adjacent tissues were obtained from patients undergoing radical prostatectomy, while prostatitis tissues and normal tissues were obtained from patients undergoing radical cystectomy. $\mathrm{PCa}$, prostate cancer; $\mathrm{BPH}$, benign prostatic hyperplasia. ${ }^{\mathrm{P}} \mathrm{P}<0.05$.

has been found in PCa (22); however, the impact of SPC24 in PCa remains to be elucidated.

The present study analyzed the TCGA database to evaluate the possible diagnostic value of SPC24 in PCa and the associations between SPC24 and clinical characteristics. The results of the TCGA dataset analysis indicated that SPC24 was highly expressed in PCa tissues compared to normal tissues. Further, results demonstrated that the high expression of SPC24 was associated with increased age, PSA value, lymph node metastasis and Gleason score, without an association with tumor level and laterality. Furthermore, analysis of a subset of clinical specimens by RT-qPCR, western blotting and immunohistochemistry demonstrated that SPC24 was significantly up-regulated in PCa tissues and BPH tissues compared to the adjacent tissues. In prostatitis tissues, SPC24 was also expressed at an increased level when compared to that in normal tissues. Future studies should include more prostate samples of patients from different nationality. The results of the present study suggested that increased SPC24 may participate in many prostatic diseases, including prostatitis, BPH and PCa.

In order to explore the potential mechanism of SPC24 and its interacting proteins in the development of prostatic diseases, bioinformatics analysis was necessary. In the present study, GO and pathway functional enrichment analysis suggested that SPC24 and its interacting proteins may participate in the cell cycle and cell division, which indicated that abnormal expression of SPC24 and its interacting proteins may interfere with the cell cycle and cell division. As loss of cell cycle control is one of the most important hallmarks of cancer (23), increased expression of SPC24 and its interacting proteins may also support the concept that there is an irregular cell cycle in $\mathrm{PCa}$ cells. Furthermore, multiple predicted pathways containing SPC24 determined from analysis in the study also hinted that 
Table III. Association between SPC24 and clinicopathological characteristics in prostate cancer tissues $(n=35)$.

SPC24 level

Clinical feature

Number of cases

Low/negative

High

P-value

Urea

Positive

Negative

0

35

Ethnicity

Han

Zhuang

Age (years)

$\leq 70$

$>70$

Smoking

Yes

No

Drinking

Yes

No

tPSA

Positive $(>4.0 \mathrm{ng} / \mathrm{ml})$

Negative $(\leq 4.0 \mathrm{ng} / \mathrm{ml})$

Creatinine

Positive

Negative

Lithic acid

Positive

Negative

$\mathrm{HCO}_{3}$ -

Positive

Negative

$\mathrm{CrCl}$

Positive ( $<80 \mathrm{ml} / \mathrm{min}$ )

Negative $(>80 \mathrm{ml} / \mathrm{min})$

Gleason stage

I+III

$\mathrm{IV}+\mathrm{V}$

26

4

31
0

22

0

13

9

15

7

13

9

4

18

4

18

22

0

2

20

6

16

6

16

15

7

14

8
1.000

0.519

1.000

1.000

0.175

0.431

$0.014^{\mathrm{a}}$

9

4

0

13

3

10

3

10

5

8

$0.035^{\mathrm{a}}$

PSA, prostate specific antigen; $\mathrm{CrCl}$, creatinine clearance. ${ }^{\mathrm{a}} \mathrm{P}<0.05$.

SPC24, cooperating with its interacting proteins, could take part in many other biological processes. Validation of these predicted pathways needs to be performed in the future. Both SPC24 and SPC25 are linked with these predicted pathways. In terms of structure of protein complex, SPC24 and SPC25 fold tightly together into a single globular entity with pseudo-2-fold symmetry in vivo $(24,25)$. In terms of protein function, SPC24 is required for meiotic kinetochore-microtubule attachment and production of euploid eggs and SPC25 is required for chromosome alignment, spindle formation and proper spindle checkpoint signaling during meiosis $(26,27)$. These studies imply that SPC24 and SPC25 are closely related, not only in structure, but also in function. In the present study, multiple predicted pathways which all contained SPC24 and SPC25suggest a close interaction between SPC24 and SPC25. However, the effect of SPC24/SPC25 complex in prostatic diseases remains unclear. The potential role of SPC24/SPC25 complex in prostatic diseases also requires further study. Dysregulation of the kinetochore may result in chromosome aneuploidy and instability, resulting from a guidance defect for proper and accurate chromosome segregation during mitosis $(28,29)$. NDC80 is known to be the core component of the NDC80 centromere complex and the protein interaction partner of SPC24 (1). In addition, high-level expression of NDC80 was found in Human PCa tissues and increased NDC80 expression can also enhance the proliferation, migration, and 
Table IV. Association between SPC24 and clinicopathological characteristics in benign prostatic hypertrophic tissues (n=40).

\begin{tabular}{|c|c|c|c|c|}
\hline \multirow[b]{2}{*}{ Clinical feature } & \multirow[b]{2}{*}{ Number of cases } & \multicolumn{2}{|c|}{ SPC24 level } & \multirow[b]{2}{*}{ P-value } \\
\hline & & Low/negative & High & \\
\hline Urea & & & & 1.000 \\
\hline Positive & 7 & 5 & 2 & \\
\hline Negative & 33 & 24 & 9 & \\
\hline Ethnicity & & & & $0.031^{\mathrm{a}}$ \\
\hline Han & 20 & 11 & 9 & \\
\hline Zhuang & 20 & 18 & 2 & \\
\hline Age (years) & & & & 0.293 \\
\hline$\leq 70$ & 19 & 12 & 7 & \\
\hline$>70$ & 21 & 17 & 4 & \\
\hline Smoking & & & & 0.286 \\
\hline Yes & 22 & 14 & 8 & \\
\hline No & 18 & 15 & 3 & \\
\hline Drinking & & & & 0.369 \\
\hline Yes & 7 & 4 & 3 & \\
\hline No & 33 & 25 & 8 & \\
\hline tPSA & & & & 0.455 \\
\hline Positive $(>4.0 \mathrm{ng} / \mathrm{ml})$ & 29 & 22 & 7 & \\
\hline Negative $(\leq 4.0 \mathrm{ng} / \mathrm{ml})$ & 11 & 7 & 4 & \\
\hline Creatinine & & & & 1.000 \\
\hline Positive & 9 & 7 & 2 & \\
\hline Negative & 31 & 22 & 9 & \\
\hline Lithic acid & & & & 1.000 \\
\hline Positive & 16 & 12 & 4 & \\
\hline Negative & 24 & 17 & 7 & \\
\hline $\mathrm{HCO}_{3-}$ & & & & 1.000 \\
\hline Positive & 20 & 15 & 5 & \\
\hline Negative & 20 & 14 & 6 & \\
\hline $\mathrm{CrCl}$ & & & & $0.020^{\mathrm{a}}$ \\
\hline Positive $(<80 \mathrm{ml} / \mathrm{min})$ & 27 & 23 & 4 & \\
\hline Negative (>80 ml/min) & 13 & 6 & 7 & \\
\hline Gleason stage & & & & 1.000 \\
\hline $\mathrm{I}+\mathrm{III}$ & 7 & 5 & 2 & \\
\hline $\mathrm{IV}+\mathrm{V}$ & 33 & 24 & 9 & \\
\hline
\end{tabular}

PSA, prostate specific antigen; $\mathrm{CrCl}$, creatinine clearance. ${ }^{\mathrm{a}} \mathrm{P}<0.05$.

invasion ability of PCa cell lines (30). It has been reported that SPC24 expression is upregulated in NDC80-overexpressing cancer cells (31). Therefore, in the present study experiments were conducted to verify NDC80 expression levels in the clinical specimens. The qPCR results showed that the level of NDC80 mRNA was higher in PCa tissues than in adjacent tissues, which was also related to expression of SPC24. The formation of proper kinetochore-microtubule attachments facilitates the procedural separation of chromosomes. The NDC80 protein is responsible for lateral attachment of microtubules, helping establish end-on attachment by indirectly interacting with the microtubule plus end through recruitment of microtubule-associated proteins (32). However, overproduction of NDC80 leads to the absorption of its interacting proteins by binding to the internal loop, blocking its interacting proteins from connecting to microtubules. This sequestration impedes mitotic progression and leads to chromosome mis-segregation, resulting in the formation of aneuploid progenies. Aneuploidy promotes tumorigenesis (33). In the present study, increased BUB1 mRNA was detected in PCa specimens. It is known that there is a surveillance system in the eukaryotic cell, the spindle assembly checkpoint (SAC), which can identify incorrectly attached or unattached kinetochores to ensure faithful chromosome segregation. The SAC consists of six components (MPH1, MAD1, MAD2, MAD3, BUB1 and BUB3). Upon SAC activation, BUB1 and other SAC components arrive 

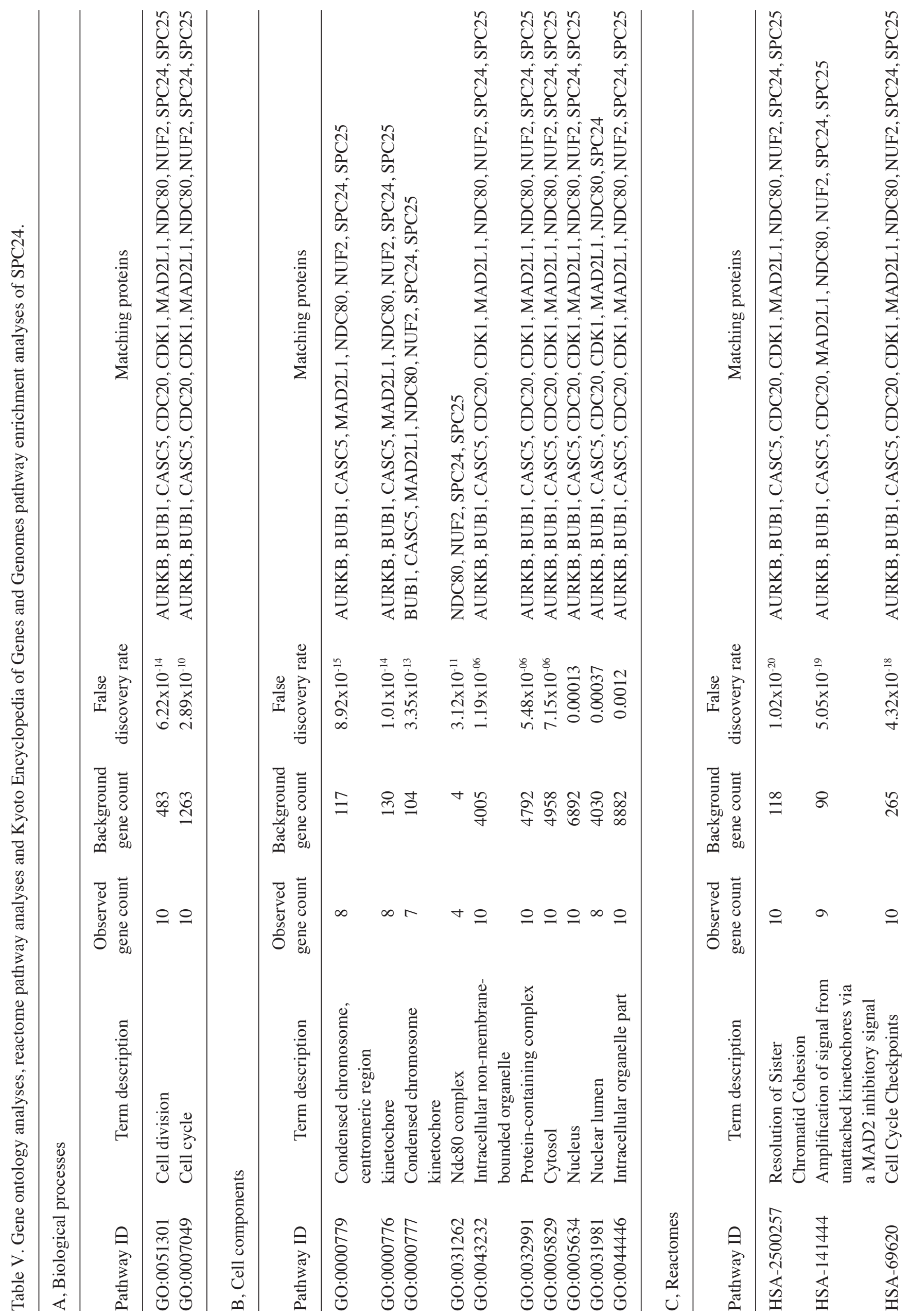


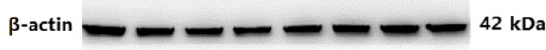

B

PCa tissues

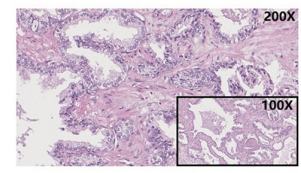

Adjacent tissues

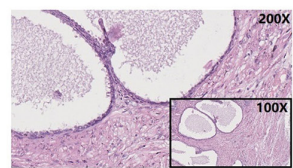

BPH tissues

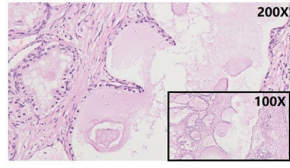

Prostatitis tissues

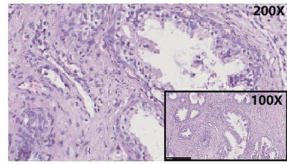

Normal tissues

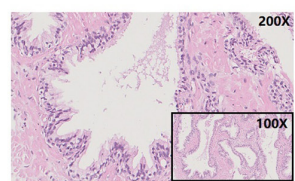

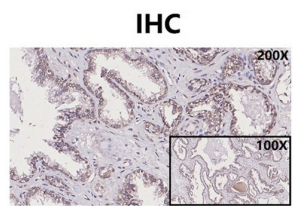
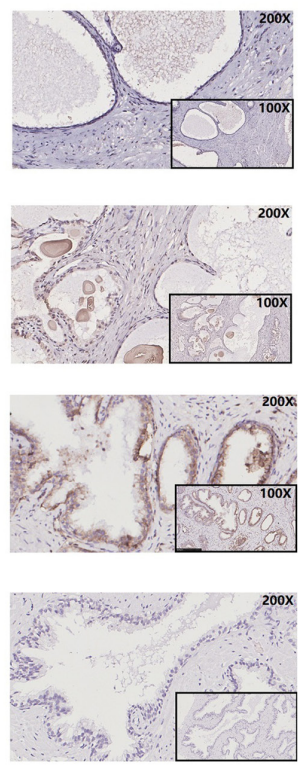

Figure 3. Protein level of SPC24 in different prostate samples. (A) Highly expressed SPC24 was found in PCa tissues compared to the adjacent tissues detected by western blotting. (B) Expression of SPC24 in different prostate samples including PCa tissues, paired-adjacent tissues, BPH tissues, prostatitis tissues, and normal tissues. Positive staining was mostly concentrated in the epithelial cells of prostate gland. $\mathrm{BPH}$, benign prostatic hypertrophy; H\&E, hematoxylin-eosin staining; IHC, immunohistochemistry; T, PCa tissue; N, paired adjacent tissues; $\mathrm{PCa}$, prostate cancer.

at the kinetochore, generating a 'wait-anaphase' signal to prevent mitotic progression until all kinetochores are properly attached to microtubules emanating from the opposite spindle poles $(34,35)$. However, when BUB1 is over-expressed, it causes mitotic errors of chromosome misalignment and lagging and resulted in near-diploid aneuploidies and tumor formation (36). These results suggest coordination among NDC80, BUB1, and SPC24 in PCa-genesis. Patients with elevated serum PSA levels are not diagnosed accurately with PCa (13). To diagnose PCa more accurately, two combined models via SPC24 mRNA were established. The AUC of two combined ROC curves was bigger than that of single SPC24, which demonstrated that combined models between SPC24 and its interacting partners could provide a discriminatory ability to diagnose PCa. At an optimal cut-off point with the maximum Youden index, the sensitivity and specificity of two combined ROC curves were different. A combined model of SPC24 and BUB1 had the highest sensitivity, while a combined model of SPC24 and NDC80 had the highest specificity. This result might be caused by different diagnostic ability of single BUB1 and NDC80, which will require further study. Compared to other previously reported PCa diagnosis markers, such as serum 
Table VI. Sensitivity and specificity to diagnose prostate cancer with SPC24 and its interacting proteins.

\begin{tabular}{lcccc}
\hline Model & AUC & Cut off & Sensitivity (\%) & Specificity (\%) \\
\hline SPC24 & 0.821 & 1.91 & 70.9 & 82.7 \\
SPC24 + BUB1 & 0.894 & 4.71 & 88.2 & 80.8 \\
SPC24 + NDC80 & 0.905 & -3.10 & 84.7 & 96.2 \\
\hline
\end{tabular}

The sensibility and specificity were obtained at an optimal cut-off point (quantified using RSEM) with the maximum Youden index. $\mathrm{PCa}$, prostate cancer; AUC, area under ROC curve; Combined models were acquired using the following algorithms (binary logistic regression): SPC24 + BUB1: Y=SPC24+BUB1*0.849/1.230; SPC24 + NDC80: Y=SPC24+NDC80*(-1.022)/1.486. AUC, area under the curve; BUB1, mitotic checkpoint serine threonine protein kinase BUB1; ROC, receiver operator characteristic.
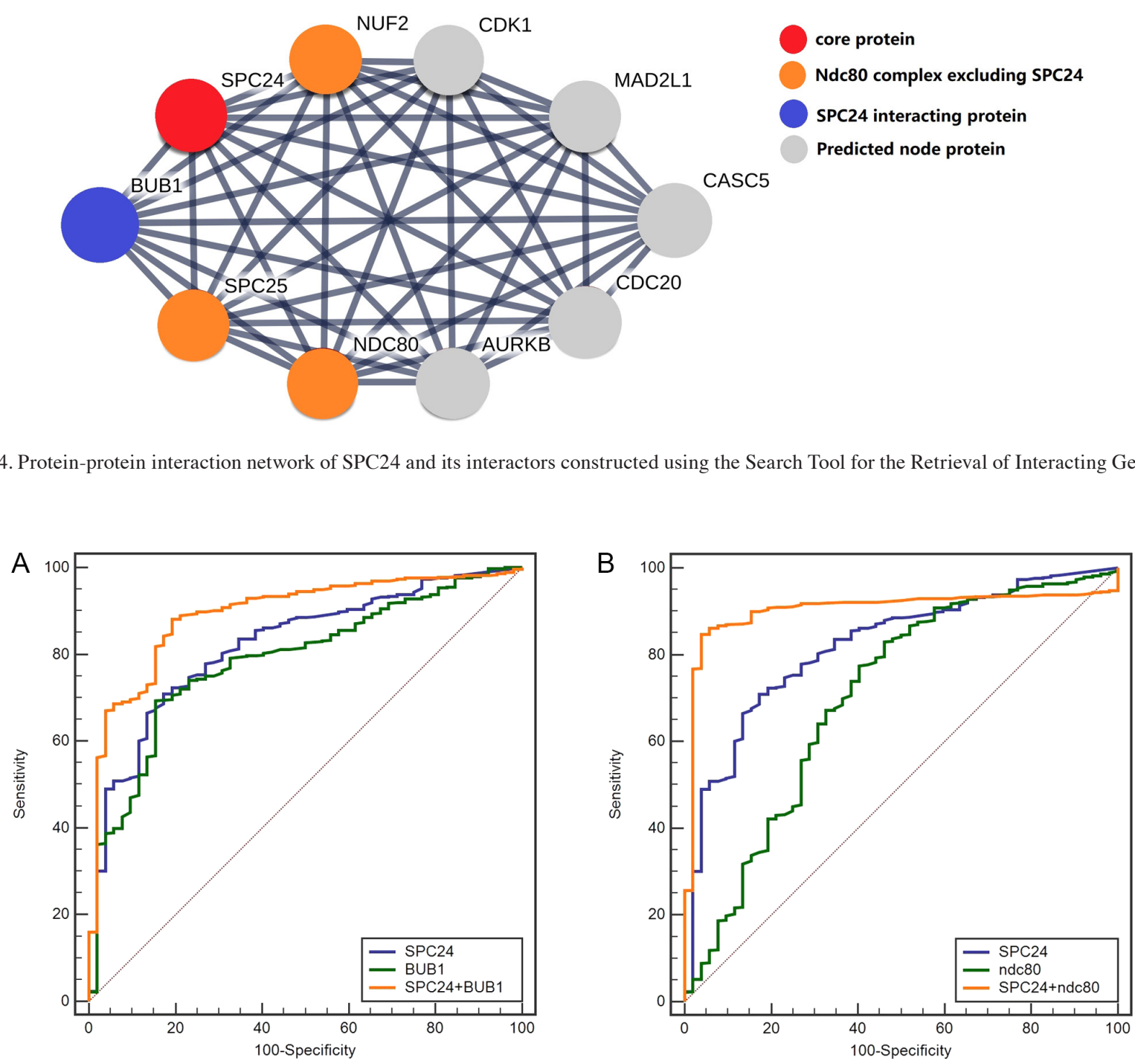

Figure 5. ROC curves of SPC24 and combined models for diagnosis of PCa. The red solid ROC curve represented combined models. (A) ROC curves of BUB1, SPC24, and combined model of BUB1 and SPC24. AUC of combined model was the biggest among three curves. (B) ROC curves of NDC80, SPC24, and combined model of NDC80 and SPC24. AUC of combined model was the biggest among three curves. AUC, area under the curve; BUB1, mitotic checkpoint serine threonine protein kinase BUB1; ROC, receiver operator characteristic.

PSA (AUC: 0.52 ; sensitivity: 58\%; specificity: $72 \%$ ), prostate cancer antigen 3 (PCA3) mRNA (AUC: 0.865; sensitivity: 94.9\%; specificity: $60.1 \%$ ), and a combined model of PSA, PCA3, human glandular kallikrein 2 (HK2) (AUC: 0.667; sensitivity: $55.8 \%$; specificity: $82.5 \%$ ) (37-39), a diagnostic model of SPC24 combining BUB1/NDC80 could provide better diagnostic ability for PCa with a bigger AUC $(>0.89)$, higher sensitivity and higher specificity (both over $80 \%$ ). To the best of our knowledge, this study was the first to demonstrate that SPC24 was significantly up-regulated in BPH, prostatitis and PCa. SPC24 and its interactors may participate in some biological processes and many reactome pathways, including 
cohesion and separation of sister chromatids. In conclusion, SPC24 may serve a carcinogenic role in the development of $\mathrm{PCa}$ and may act as a new diagnostic and potential therapeutic target for PCa. The diagnostic and prognostic values of SPC24 and its possible therapeutic applications are worthy of further investigation.

\section{Acknowledgements}

Prof. Jian Qin from school of public health, Guangxi Medical University was invited to check the statistical methods in our study.

\section{Funding}

This work was supported by the National Natural Science Foundation of China (grant nos. 81560130 and 21505025) and the Guangxi Nature Science Foundation (grant nos. 2018GXNSFAA281033, 2016GXNSFDA380010 and 2013GXNSFGA019005).

\section{Availability of data and materials}

The datasets used and/or analyzed during the present study are available from the corresponding author on reasonable request.

\section{Authors' contributions}

CL and XY made substantial contributions to conception and design and agreed to be accountable for all aspects of the work in ensuring that questions related to the accuracy or integrity of any part of the work were appropriately investigated and resolved. SC, XW and SZ wrote and revised the manuscript, performed the experiments and analyzed and interpreted scientific data. SC and SQ confirmed the authenticity of all the raw data. HL, SQ, JL and WJ performed IHC, qPCR and western blot analysis. MS, YT, HL, WS and SL participated in data collection and analysis. All authors read and approved the final manuscript.

\section{Ethics approval and consent to participate}

The present study was approved by the Ethics Committee of The First Affiliated Hospital of Guangxi Medical University (Nanning, China).

\section{Patient consent for publication}

Not applicable.

\section{Competing interests}

The authors declare that they have no competing interests.

\section{References}

1. DeLuca JG and Musacchio A: Structural organization of the kinetochore-microtubule interface. Curr Opin Cell Biol 24: 48-56, 2012.

2. Cheeseman IM and Desai A: Molecular architecture of the kinetochore-microtubule interface. Nat Rev Mol Cell Biol 9: 33-46, 2008
3. Wilson-Kubalek EM, Cheeseman IM, Yoshioka C, Desai A and Milligan RA: Orientation and structure of the Ndc80 complex on the microtubule lattice. J Cell Biol 182: 1055-1061, 2008.

4. Dominguez-Brauer C, Thu KL, Mason JM, Blaser H, Bray MR and Mak TW: Targeting mitosis in cancer: Emerging strategies. Mol Cell 60: 524-536, 2015.

5. Ma L, McQueen J, Cuschieri L, Vogel J and Measday V: Spc24 and Stu2 promote spindle integrity when DNA replication is stalled. Mol Biol Cell 18: 2805-2816, 2007.

6. Bharadwaj R and Yu H: The spindle checkpoint, aneuploidy, and cancer. Oncogene 23: 2016-2027, 2004.

7. Siegel RL, Miller KD and Jemal A: Cancer statistics, 2018. CA Cancer J Clin 68: 7-30, 2018.

8. Nelson WG, De Marzo AM and Isaacs WB: Prostate cancer. N Engl J Med 349: 366-381, 2003.

9. Gutiérrez-González E, Castelló A, Fernández-Navarro P, Castaño-Vinyals G, Llorca J, Salas D, Salcedo-Bellido I, Aragonés N, Fernández-Tardón G, Alguacil J, et al: Dietary zinc and risk of prostate cancer in Spain: MCC-Spain Study. Nutrients 11: 18, 2018

10. Hung SC, Lai SW, Tsai PY, Chen PC, Wu HC, Lin WH and Sung FC: Synergistic interaction of benign prostatic hyperplasia and prostatitis on prostate cancer risk. Br J Cancer 108: 1778-1783, 2013.

11. Dai X, Fang X, Ma Y and Xianyu J: Benign prostatic hyperplasia and the risk of prostate cancer and bladder cancer: A meta-analysis of observational studies. Medicine (Baltimore) 95: e3493, 2016.

12. Boorjian SA, Eastham JA, Graefen M, Guillonneau B, Karnes RJ, Moul JW, Schaeffer EM, Stief C and Zorn KC: A critical analysis of the long-term impact of radical prostatectomy on cancer control and function outcomes. Eur Urol 61: 664-675, 2012.

13. Sharma N and Baruah MM: The microRNA signatures: Aberrantly expressed miRNAs in prostate cancer. Clin Transl Oncol 21: 126-144, 2019.

14. Zhou J, Pei Y, Chen G, Cao C, Liu J, Ding C, Wang D, Sun L, $\mathrm{Xu}$ P and Niu G: SPC24 Regulates breast cancer progression by PI3K/AKT signaling. Gene 675: 272-277, 2018.

15. Zhou J, Yu Y, Pei Y, Cao C, Ding C, Wang D, Sun L and Niu G: A potential prognostic biomarker SPC24 promotes tumorigenesis and metastasis in lung cancer. Oncotarget 8: 65469-65480, 2017.

16. Yin H, Meng T, Zhou L, Chen H and Song D: SPC24 is critical for anaplastic thyroid cancer progression. Oncotarget 8: 21884-21891, 2017.

17. Sheng J, Yin M, Sun Z, Kang X, Liu D, Jiang K, Xu J, Zhao F, Guo Q and Zheng W: SPC24 promotes osteosarcoma progression by increasing EGFR/MAPK signaling. Oncotarget 8: 105276-105283, 2017.

18. Livak KJ and Schmittgen TD: Analysis of relative gene expression data using real-time quantitative PCR and the 2(-Delta Delta C(T)) method. Methods 25: 402-408, 2001.

19. Tokuzumi A, Fukushima S, Miyashita A, Nakahara S, Kubo Y, Yamashita J, Harada M, Nakamura K, Kajihara I, Jinnin M and Ihn H: Cell division cycle-associated protein 1 as a new melanoma-associated antigen. J Dermatol 43: 1399-1405, 2016.

20. Obara W, Sato F, Takeda K, Kato R, Kato Y, Kanehira M, Takata R, Mimata H, Sugai T, Nakamura Y and Fujioka T: Phase I clinical trial of cell division associated 1 (CDCA1) peptide vaccination for castration resistant prostate cancer. Cancer Sci 108: 1452-1457, 2017.

21. McCleland ML, Kallio MJ, Barrett-Wilt GA, Kestner CA, Shabanowitz J, Hunt DF, Gorbsky GJ and Stukenberg PT: The vertebrate Ndc80 complex contains Spc24 and Spc25 homologs, which are required to establish and maintain kinetochoremicrotubule attachment. Curr Biol 14: 131-137, 2004.

22. Cui F, Hu J, Fan Y, Tan J and Tang H: Knockdown of spindle pole body component 25 homolog inhibits cell proliferation and cycle progression in prostate cancer. Oncol Lett 15: 5712-5720, 2018.

23. Hanahan D and Weinberg RA: The hallmarks of cancer: The next generation. Cell 144: 646-674, 2011.

24. Wei RR, Sorger PK and Harrison SC: Molecular organization of the Ndc80 complex, an essential kinetochore component. Proc Natl Acad Sci USA 102: 5363-5367, 2005.

25. Wei RR, Schnell JR, Larsen NA, Sorger PK, Chou JJ and Harrison SC: Structure of a central component of the yeast kinetochore: The Spc24p/Spc25p globular domain. Structure 14: 1003-1009, 2006.

26. Sun SC, Lee SE and Xu YN: Perturbation of SPC25 expression affects meiotic spindle organization, chromosome alignment and spindle assembly checkpoint in mouse oocytes. Cell cycle 9: 4552-4559, 2010 
27. Zhang T, Zhou Y, Wang HH, Meng TG, Guo L, Ma XS, Shen W, Schatten H and Sun QY: SPC24 is required for meiotic kinetochore-microtubule attachment and production of euploid eggs. Oncotarget 7: 71987-71997, 2016.

28. Charters GA, Stones CJ, Shelling AN, Baguley BC and Finlay GJ: Centrosomal dysregulation in human metastatic melanoma cell lines. Cancer Genet 204: 477-485, 2011.

29. Tomonaga T, Matsushita K, Ishibashi M, Nezu M, Shimada H, Ochiai T, Yoda $\mathrm{K}$ and Nomura F: Centromere protein $\mathrm{H}$ is up-regulated in primary human colorectal cancer and its overexpression induces aneuploidy. Cancer Res 65: 4683-4689, 2005

30. Wang H, Gao X, Lu X, Wang Y, Ma C, Shi Z, Zhu F, He B, Xu C and Sun Y: The mitotic regulator Hecl is a critical modulator of prostate cancer through the long non-coding RNA BX647187 in vitro. Biosci Rep 35: e00273, 2015.

31. Huang LY, Chang CC, Lee YS, Chang JM, Huang JJ, Chuang SH Kao KJ, Lau GM, Tsai PY, Liu CW, et al: Activity of a novel Hecl-targeted anticancer compound against breast cancer cell lines in vitro and in vivo. Mol Cancer Ther 13: 1419-1430, 2014.

32. Chmielewska AE, Tang NH and Toda T: The hairpin region of Ndc80 is important for the kinetochore recruitment of Mph1/MPS1 in fission yeast. Cell Cycle 15: 740-747, 2016.

33. Tang NH and Toda T: MAPping the Ndc80 loop in cancer: A possible link between $\mathrm{Ndc} 80 / \mathrm{Hecl}$ overproduction and cancer formation. Bioessays 37: 248-256, 2015.

34. Musacchio A: The molecular biology of spindle assembly checkpoint signaling dynamics. Curr Biol 25: R1002-R1018, 2015.
35. Lara-Gonzalez P, Westhorpe FG and Taylor SS: The spindle assembly checkpoint. Curr Biol 22: R966-R980, 2012.

36. Ricke RM, Jeganathan KB and van Deursen JM: Bub1 overexpression induces aneuploidy and tumor formation through Aurora B kinase hyperactivation. J Cell Biol 193: 1049-1064, 2011.

37. Marks LS, Fradet Y, Deras IL, Blasé A, Mathis J, Aubin SM, Cancio AT, Desaulniers M, Ellis WJ, Rittenhouse H and Groskopf J: Pca3 molecular urine assay for prostate cancer in men undergoing repeat biopsy. Urology 69: 532-535, 2007.

38. Merola R, Tomao L, Antenucci A, Sperduti I, Sentinelli S, Masi S, Mandoj C, Orlandi G, Papalia R, Guaglianone S, et al: $\mathrm{Pca} 3$ in prostate cancer and tumor aggressiveness detection on 407 high-risk patients: A national cancer institute experience. J Exp Clin Cancer Res 34: 15, 2015.

39. Mao Z, Ji A, Yang K, He W, Hu Y, Zhang Q, Zhang D and Xie L: Diagnostic performance of PCA3 and hK2 in combination with serum PSA for prostate cancer. Medicine (Baltimore) 97: e12806, 2018.

This work is licensed under a Creative Commons Attribution-NonCommercial-NoDerivatives 4.0 International (CC BY-NC-ND 4.0) License. 